\title{
The APA Style Converter: A Web-based interface for converting articles to APA style for publication
}

\author{
PING LI and KRYSTAL CUNNINGHAM \\ University of Richmond, Richmond, Virginia
}

\begin{abstract}
The APA Style Converter is a Web-based tool with which authors may prepare their articles in APA style according to the APA Publication Manual (5th ed.). The Converter provides a user-friendly interface that allows authors to copy and paste text and upload figures through the Web, and it automatically converts all texts, references, and figures to a structured article in APA style. The output is saved in PDF or RTF format, ready for either electronic submission or hardcopy printing.
\end{abstract}

The APA style is a set of guidelines provided by the American Psychological Association (2001) to authors for preparing their articles for publication. These guidelines are specified in the APA Publication Manual (5th ed.), and the manual contains important information about writing scientific articles in a consistent and coherent style. The guidelines have been widely adopted by researchers in the social and behavioral sciences, including psychology. Many scientific journals also require authors to submit articles in APA style.

Although the APA style has widespread use in the scientific community, several factors hinder authors from accurate use of the style. First, many authors find it difficult to keep track of all the details specified in the manual. For example, when asked, few researchers are certain about (1) whether the footnotes section should come before or after the author note, (2) whether the appendices should come before or after the tables, or (3) what a page header is, how it is different from a running head, and how these head and headers should be laid out on the title page. Second, some authors who are familiar with earlier versions of the APA style might not have kept up with the newest changes in the current version, such as the abolition of underlines and the citation for Internet resources. Third, researchers from countries other than those in North America and Europe are relatively unfamiliar with the APA style, either because it is not part of their training or because writing conventions and publication guidelines for their native languages differ from the APA style.

Preparation of this article and the work reported here were made possible by Grant BCS-0131829 from the National Science Foundation to P.L. We thank Megan Kuhn and Sara Sepanski for their assistance in the development of the teaching resource Web site for Guidelines on Writing in APA Style. We also thank Xiaowei Zhao and Shuxia Liu for making the Chinese version of the APA Style Converter. Updated versions of the Converter are available at http://cogsci.richmond.edu/APAconverter/ main.html. Please address correspondence to P. Li, Department of Psychology, University of Richmond, Richmond, VA 23173 (e-mail: pli@richmond.edu).
Yet more and more researchers find it necessary to deal with the APA style: The style is widely used in many disciplines, and the journals to which they submit articles require it. Researchers from other countries are also under increasing pressure to publish in international journals that may require the APA style.

Given these considerations, we have developed a userfriendly tool that allows authors to convert their articles to APA style through a Web-based interface: The APA Style Converter (henceforth, the Converter). The Converter differs from other commercialized software, such as the APA-Style Helper, which often requires authors to input, page by page, the title, the abstract, and other sections in a tedious manner. Instead, authors using the Converter can cut and paste all text into the dialogue boxes and can upload figures and images all at once. The Converter automatically handles the formatting details, such as the placement of information on the title page, the page numbers, the placement of section headings, the ordering of sections, and so on (see details below). However, the Converter is not designed for authors to write their articles on line, but for authors who have completed the article in writing (in whichever format and style) and are ready to convert the article to APA style for submission. For example, this document has been generated with the Converter by the authors, following the procedure discussed below.

\section{Overview of the Converter}

The main page. To access the Converter, users point their browser to the main page of the Converter: http:// cogsci.richmond.edu/APAconverter/main.html. The Converter currently works optimally with Internet Explorer (Version 6.0 or above) or Netscape (Version 7.0 or above). The main page is a gateway to several other pages that contain important information or provide functions of the Converter, as is shown in Figure 1.

1. Users can decide whether they want to stay on the current version in English or whether they want to switch to the Chinese version or the Spanish version. To switch 


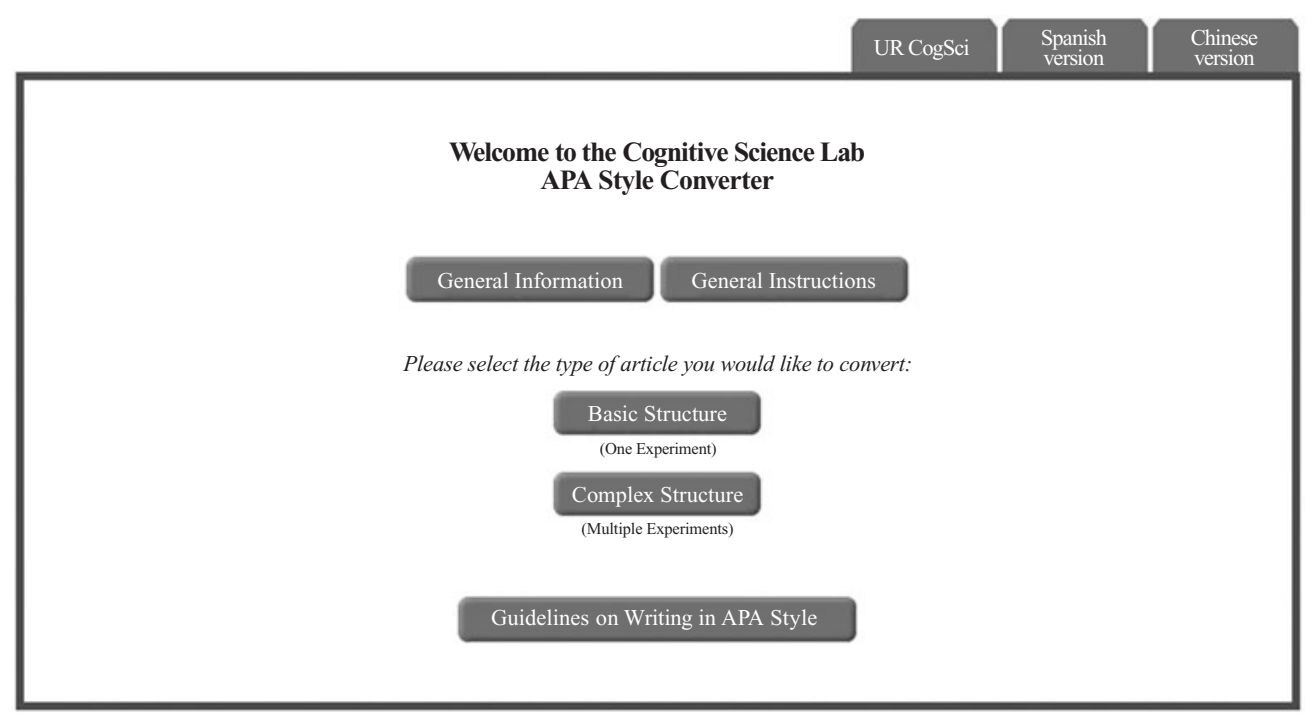

Figure 1. The main page of the APA Style Converter.

to other language versions, they simply click on the links at the upper right hand corner of the page. The Chinese version and the Spanish version provide exactly the same interface as the English version, and the only difference between these versions is in the language of instruction.

2 . Users can point to General Information for a detailed description of the Converter, which is this article in PDF format.

3. Users need to read the General Instructions page that provides details on how to cut and paste, how to convert and upload images, and how to treat italics, footnotes, and statistical symbols. For details, please see the discussion in the Special Formats and Symbols section below.

4. Users have to decide on the article type on this page: whether their article has the basic structure (i.e., contains only a single experiment) or the complex structure (i.e., contains multiple experiments). Depending on the article type selected, the Converter will yield different formats according to the APA style.

5. Users can read more details on the APA style by clicking on the Guidelines on Writing in APA Style link. This will bring the user to a teaching resource Web site that provides summary information on how to write articles in APA style, including sample articles and other useful links.

The Basic Structure page. Once users decide on the type of article (single experiment or multiple experiments), they are ready to enter text, copy and paste text, and upload images. Let's illustrate this process with the basic structure page.

1. Filename. Users need to choose a filename for converting the document on the Web. This filename also serves to keep track of the various parts of the text and images and, hence, needs to be entered whenever an image is uploaded (see the description below).
2. Title page. Users should enter the title, the running head, and the page header in the corresponding dialogue boxes. Next, they should enter the institutional affiliations and author name or names, following the format $<$ Affiliation: Author $>$. In cases with multiple authors from the same institution, the format is <Affiliation: Author1, Author2>. In cases with multiple authors from different institutions, the format is <Affiliation 1: Author1; Affiliation2: Author2>. On the basis of the entered information, the Converter makes the layout of the title page, with title, author names, affiliations, running head, and page header appropriately displayed according to APA style. Starting from the Title dialogue box, the user can also access the help pages by clicking on the help icon above the boxes (see the Help Pages section below). An example of the basic structure page is shown in Figure 2.

3. Abstract. The user simply copies and pastes the abstract from the original file to this box. Note that the user does not need to type the heading Abstract in the text, and this holds for all major sections of the article discussed below.

4. Introduction, method, results, and discussion sections. For each of these sections, the user copies and pastes the original text into the dialogue box. There are potential problems that the user should be aware of during copying and pasting. For example, when copying from a word processor and pasting to a browser, a number of formatting details may be lost. In particular, if the user has used special symbols, special characters, and other special formatting patterns that are not recognized by the browser, these may not come out correctly (see the Special Formats and Symbols section below; in such cases, the RTF option should be used).

5. References. APA imposes a set of conventions for reference formatting for consistency in reference cita- 


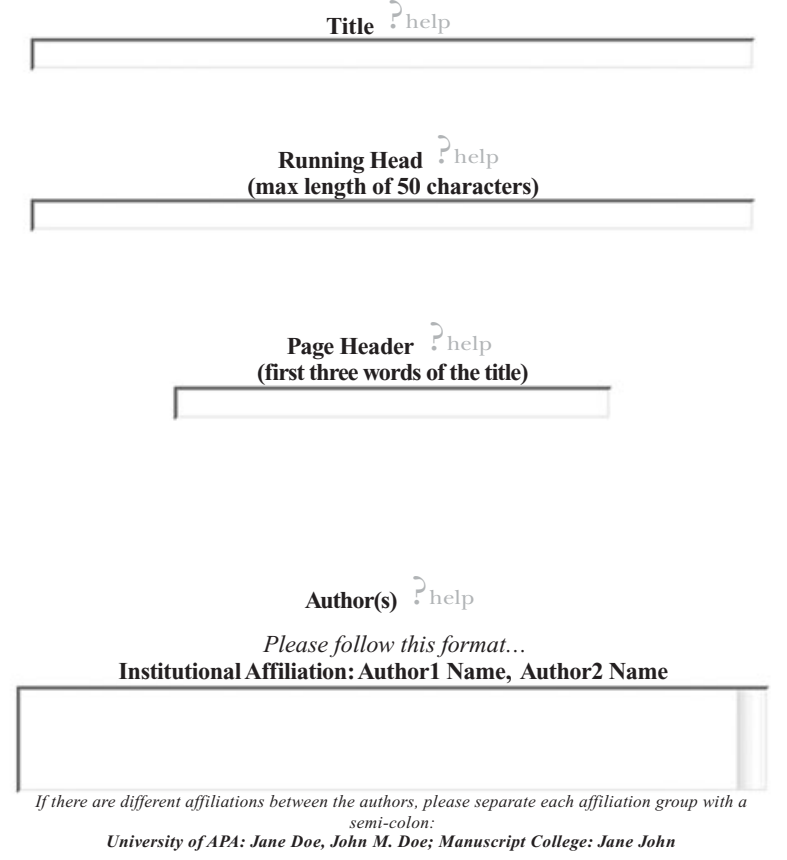

Figure 2. A portion of the Basic Structure page.

tions. However, these complex conventions escape the memories of most authors, especially regarding the details on the use of spaces, parentheses, italics, periods, and upper- and lowercase characters for various types of reference items. Thus, the Converter provides an interface that allows the user to enter author names, article titles, and other publication details in an itemized fashion through dialogue boxes, which can then be automatically converted to the APA reference style. Figure 3 illustrates an example for journal articles. Alternatively, if users think that their reference section fully conforms to the APA style and do not want to go through itemized entry, they can save the reference section as a JPEG image and upload it through the Converter. Instructions on how to convert formatted text to a JPEG image are provided under the general instructions page.

6. Other sections. Users can copy and paste their acknowledgments and footnotes to the author note and footnotes dialogue boxes. If there are appendices, tables, and figures, these should be saved as JPEG images and uploaded. For tables and figures, the user should upload only the table or figure per se and provide the title of tables and figure captions through the Converter's table and figure upload pages.

When information on the above six portions is provided, the user is now ready to hit the Convert to APA button. The conversion process will automatically assemble all sections that contain the relevant information (text and images) provided by the user, put them in the correct order according to the APA style, and then generate a PDF or RTF file as output on the user's desktop. The PDF file conforms fully to the APA style and cannot be directly modified. Minor modifications are possible with Adobe Acrobat (the professional version), but if major changes are needed, the user should go back to the Converter interface, change the details there, and start the conversion process over again (the main text and images should remain on the browser if the user has not closed the browser). The RTF file, however, can be modified in whichever way the user wants and can be saved as a Word document. Thus, the RTF option is more suitable for articles that are not final.

The Complex Structure page. The Complex Structure page has the same properties and functions as the basic structure page discussed above. The difference between the two is that the Complex Structure page can accommodate more than one experiment (up to three experiments in the current version) and, consequently, the headings and subheadings of the article will be structured differently from those in the basic structure article, according to APA style.

The help pages. A useful feature of the Converter is that for each section, the Converter provides a help page that gives summary information on how to write the particular section in APA style and what aspects of this section need particular attention. Users can click on the help icon to get to these pages. The pages are the same pages as those from the teaching resource Web site that users 


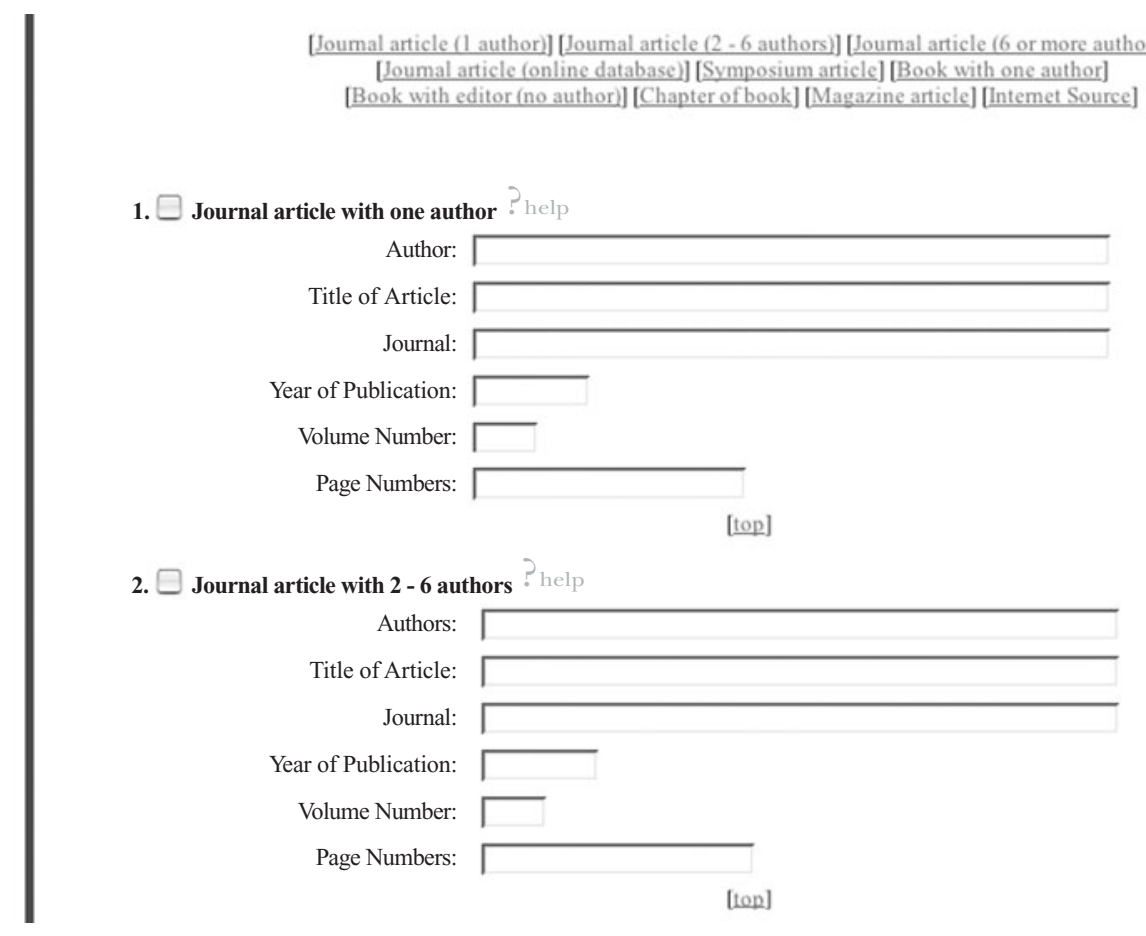

Figure 3. A portion of the References page for itemized entry.

can access through the Converter's main page (Guidelines on Writing in APA Style). This Web site has been developed for a research method class, and the first author uses it to teach undergraduate psychology students at the University of Richmond how to write articles in proper APA style (http://www.richmond.edu/ pli/teaching/ psy200/apastyle/apastyle.html). An example page is given in Figure 4.

Special formats and symbols. The Converter automatically handles formatting details according to the APA style, but the user should be aware that some formats may not be pasted correctly from a word processor onto the browser. In general, the APA style requires few special symbols and formats, and the user should check whether they are essential to the article and, if so, to what extent the browser under use allows them to be displayed correctly.

In order to preserve the special formats or special symbols, the Converter requires users to pay attention to the following.

1. Block or indented quotes (i.e., 0.5 in. from the left margin) should be surrounded by \#BQ and \#bq, each on a separate line.

2. Italics may be marked by $<\mathrm{i}>$ and $</ \mathrm{i}>$, with the italicized word in between (not on separate lines). Note that the Converter automatically italicizes common statistical symbols for the basic structure articles (including $p, S D$, $M, N, F, t)$ and italicizes the main subheadings in the basic structure (Participants, Subjects, Procedure, Materials, and Apparatus) and in the complex structure (Method, Results, and Discussion).
3. Centering of headings or subheadings should be marked by brackets, [ and ], with the centered material in between the brackets.

4. Footnotes should be used with caution, since their use is generally discouraged, both in the APA style and by our Converter (the Converter can handle footnotes only in the RTF format, with \{Footnote \#\} inserted in the appropriate text).

5. Other symbols and characters should be used with caution, and the user should check whether the browser has the necessary fonts to preserve the symbols or characters. If many special symbols and characters are used, the user should convert the article to RTF format and then insert the symbols in the RTF output file.

Given the need to preserve formatting details, users are encouraged to convert any specially formatted materials (e.g., appendices, figures, formatted references, and tables) to image files and then upload the images to the Converter. This can be done easily by copying the materials from a word processor (e.g., Word) to a photo editor (e.g., Photoshop). A $450 \times 550$ pixel (width $\times$ height) layout is recommended for each converted image so that it fits easily in a page. Alternatively, one can use the RTF output option, and insert figures, tables, appendices, and images into the RTF output file.

\section{Conclusions and Future Developments}

Stylistic conventions such as the APA style are designed to help authors achieve consistency, coherence, and clarity in writing. Yet the amount of details from these conventions represents a heavy burden on the part 


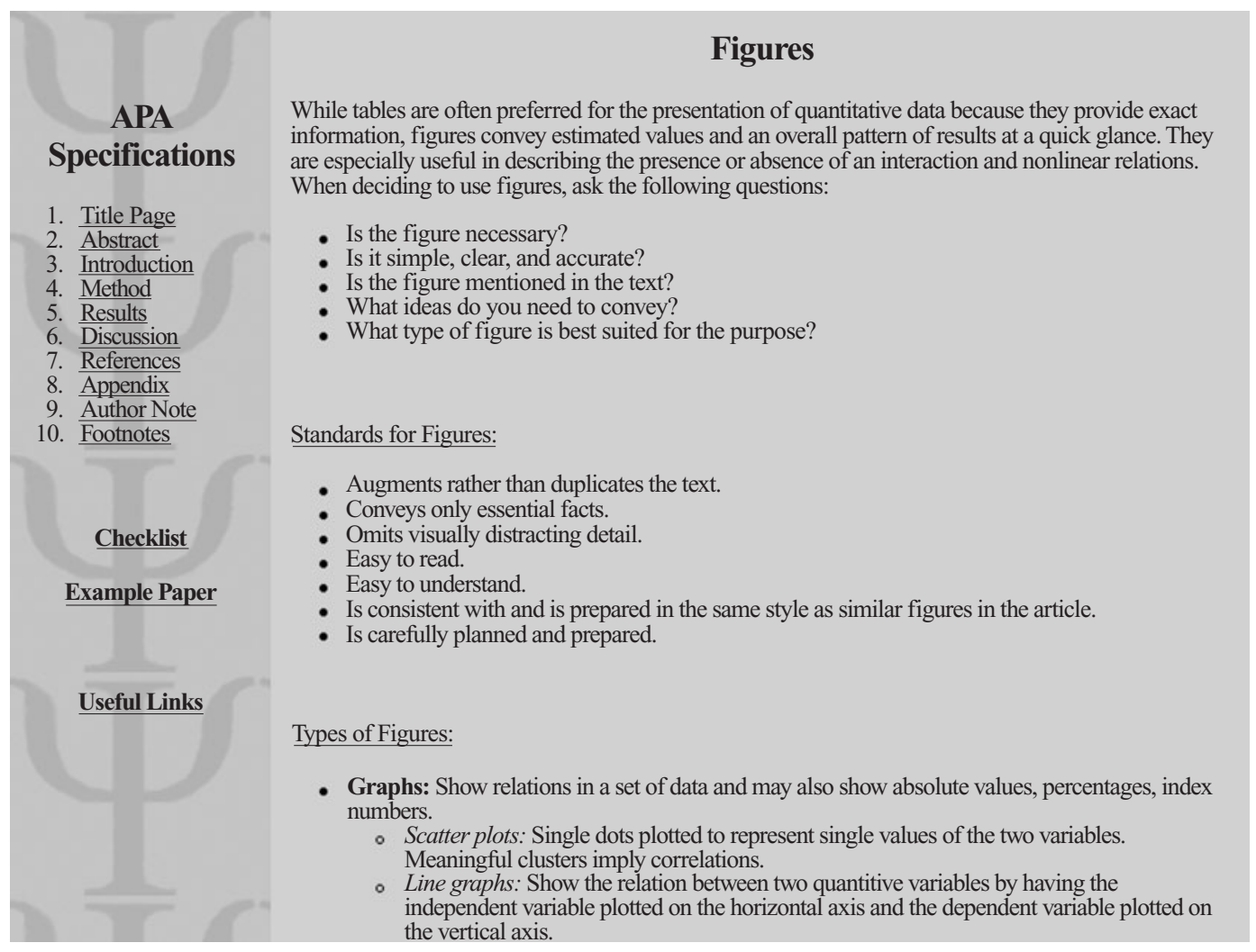

Figure 4. A help page for figures in Guidelines on Writing in APA Style.

of the author. The APA Style Converter is designed to ease some of this burden by allowing authors to automatically transform their manuscripts to the APA style through a Web interface. Thus, authors from a variety of disciplines can use the Converter to achieve or check for style consistency for their manuscripts. In addition, editors of journals that require the APA style can also use the Converter as a gateway for authors to submit their manuscripts in proper format.

The Converter in its current form represents only a beginning in the effort to automate the process of writing in style. Future developments of this work can aim at a more user-friendly interface that can automatically handle detailed formatting needs, such as special symbols and characters. In addition, through the use of database tools, the Converter should be able to allow spreadsheet-type entry of tables, full APA reference conventions, user- defined heading and subheading structures, and save-andcontinue functions that would allow users to write the article on line. If such automated efforts are successful with the APA style, there is every reason to believe that they can be generalized to deal with other stylistic conventions, such as those in the MLA (Modern Language Association) manual, the Chicago Manual, Turabian, and the CBE (Council of Biology Editors) style manual.

\section{REFERENCE}

American Psychological Association (2001). Publication manual of the American Psychological Association (5th ed.). Washington, DC: Author.

(Manuscript received October 25, 2004 accepted for publication January 17, 2005.) 